\title{
Further phenotypic features and two novel $P O C 1 A$ variants in a patient with SOFT syndrome: A case report
}

\author{
SONGTING LI ${ }^{1}$, YAN ZHONG ${ }^{1}$, YONGJIA YANG ${ }^{2}$, SIPING HE $^{3}$ and WENJUAN HE ${ }^{1}$ \\ ${ }^{1}$ Children's Healthcare Institute, Hunan Children's Hospital, University of South China; ${ }^{2}$ The Laboratory of \\ Genetics and Metabolism, Hunan Children's Research Institute, Hunan Children's Hospital, University of South China; \\ ${ }^{3}$ Department of Radiology, Hunan Children's Hospital, University of South China, Changsha, Hunan 410007, P.R. China
}

Received November 27, 2020; Accepted April 9, 2021

DOI: $10.3892 / \mathrm{mmr} .2021 .12133$

\begin{abstract}
Short stature, onychodysplasia, facial dysmorphism and hypotrichosis (SOFT) syndrome is a rare autosomal recessive disease caused by POC1 centriolar protein A (POC1A) pathogenic variants. However, knowledge of genotypic and phenotypic features of SOFT syndrome remain limited as few families have been examined; therefore, the clinical identification of SOFT syndrome remains a challenge. The aim of the present case report was to investigate the genetic cause of this syndrome in a patient with a short stature, unusual facial appearance, skeletal dysplasia and sparse body hair. Giemsa banding and exome sequencing were performed to investigate the genetic background of the family. Spiral computed tomography and magnetic resonance imaging were used for investigating further phenotypic features of the patient. Exome sequencing identified that POC1A had two compound heterozygous variants, namely c.850_851insG and c.593_605delGTGGGACGTGCAT, which, to the best of our knowledge, have not been reported elsewhere. Novel phenotypes were also identified as follows: i) Metaphyseal dysplasia was alleviated (and/or even disappeared) with age; ii) the density of the femoral neck was uneven and the hyperintensity signal of the metaphysis was stripe-like. Thus, the present case report expands the knowledge regarding phenotypic and genotypic features of SOFT syndrome.
\end{abstract}

Correspondence to: Dr Yan Zhong, Children's Healthcare Institute, Hunan Children's Hospital, University of South China, 86 Ziyuan Road, Changsha, Hunan 410007, P.R. China

E-mail: zhongyan@163.com

Dr Yongjia Yang, The Laboratory of Genetics and Metabolism, Hunan Children's Research Institute, Hunan Children's Hospital, University of South China, 86 Ziyuan Road, Changsha, Hunan 410007, P.R. China

E-mail: yongjia727@aliyun.com

Key words: short stature, onychodysplasia, facial dysmorphism and hypotrichosis syndrome, POC1 centriolar protein A, novel variants, imaging

\section{Introduction}

In 2012, Shalev et al (1) described a novel syndrome in two unrelated consanguineous Arab families with multiple members exhibiting severe short stature, skeletal dysplasia, unusual facial features, brachydactyly and hypotrichosis. Shaheen et al (2) and Sarig et al (3) identified that the biallelic variants of POC1 centriolar protein A (POC1A) cause short stature, onychodysplasia, facial dysmorphism and hypotrichosis (SOFT) syndrome. Prevalence of SOFT syndrome remains unclear. Thus far, only 13 families, with 11 POClA pathogenic variants identified in 27 patients, have been reported worldwide (4). All of these families were sporadically distributed in different countries (Table SI), including Saudi Arabia, Turkey, Iran and Oman (Middle East); Spain, Monaco and Italy (Europe); South Korea (East Asia); and Chile (South America).

Patients with SOFT syndrome have been reported to exhibit a wide range of variable phenotypes, including severe pre- and postnatal growth retardation, onychodysplasia, hypotrichosis, facial deformities (such as long triangular face, prominent forehead and pointed chin), skeletal abnormalities (such as short long bones and irregular changes in metaphysis, short femoral neck, and delayed ossification of carpal and vertebral bones), as well as a poor response to recombinant human growth hormone (rhGH) therapy (1,3,5-9). An atypical type of SOFT syndrome has also been reported in two other unrelated families, where affected members also show insulin resistance caused by dyslipidemia $(10,11)$.

Data regarding SOFT syndrome are limited due to the scarcity and phenotypic diversity of this novel entity, and its clinical identification remains a challenge. For this reason, genotypic and phenotypic features of SOFT syndrome require further investigation. In the present case report, a Chinese patient with SOFT syndrome is presented. Exome sequencing identified compound heterozygous variants of POClA. Furthermore, follow-up radiological imaging at various ages revealed several novel phenotypic findings relating to SOFT syndrome.

\section{Case report}

General information. A female patient was referred to Hunan Children's Hospital (Changsha, China) for growth retardation 
on February 11, 2019. The patient was born via spontaneous vaginal delivery at full term and had a birth weight of $1.8 \mathrm{~kg}$ ( $<3$ rd percentile) and a birth height of $40 \mathrm{~cm}(<3 \mathrm{rd}$ percentile). Her parents (both Chinese Han) were healthy and from two unrelated families. Her father was 36 years old and her mother was 35 years old. Her father's height was $175 \mathrm{~cm}(50-75 \mathrm{th}$ percentile) and her mother's height was $155 \mathrm{~cm}(10-25 \mathrm{th}$ percentile). When the patient was aged 7 years and 8 months, her height was $102.5 \mathrm{~cm}$ ( $<3 \mathrm{rd}$ percentile) and her weight was $17.5 \mathrm{~kg}$ ( $<3$ rd percentile). She had an unusual facial appearance, with a triangular face, prominent forehead and irregularly positioned teeth (Fig. 1A and B). Her fingers were stubby and with nail hypoplasia (Fig. 1C), and her body hair was sparse (Fig. 1D). Her intelligence quotient was 78 according to the Chinese Wechsler Intelligence Scale for Children (12). The patient underwent rhGH therapy for 6 months and her height increased by $4.7 \mathrm{~cm}$. Over the subsequent 3 months, her height increased by only $0.5 \mathrm{~cm}$ and the rhGH therapy was discontinued.

No abnormalities were observed in laboratory tests, which included complete blood count, urine analysis, vitamin D level, blood sugar, thyroid function level, insulin-like growth factor (IGF) binding protein 3, IGF1 and growth hormone stimulation tests. Female patients with Turner syndrome (sex chromosome aneuploidy) can exhibit a variety of developmental problems, including short height; therefore, Giemsa (GTG) banding was performed on the patient, but her karyotype was identified to be normal (46 XX; Fig. S1A and B). Ultrasound indicated left renal pelvis separation and mild tricuspid regurgitation (data not shown).

Ethics statement. Parents/guardians of both the patient and the matched control, as well as family members whose data are presented in this case report, provided written informed consent for publication of the data and associated images in the present case report. This study and all the data described in the paper were approved by the Ethics Committee of the Hunan Children's Hospital (approval no. HCHLL-020-16).

GTG banding. Peripheral venous blood was collected in a vacutainer vial containing sodium heparin. Slides were prepared using standard cytogenetic methods. GTG banding at a 400- to a 550-band level was performed in accordance with the standard laboratory protocol (13). Two different cultures corresponding to two different series of the slides from the sample were separately prepared and analyzed. At least 40 metaphases were examined for the proband of the family. For the second round of GTG banding evaluation, 100 metaphases were evaluated for the proband.

Next generation sequencing. All three family members were subjected to exome sequencing as described previously (13). Briefly, genomic DNA from three family members was isolated from the peripheral blood using a DNA Isolation Kit (Blood DNA Kit V2; cat. no. CW2553; CoWin Biosciences). The genomic DNA was quantified using the Qubit ${ }^{\mathrm{TM}}$ dsDNA HS Assay Kit (cat. no. Q32851; Invitrogen; Thermo Fisher Scientific, Inc.). The DNA of each patient was fragmented into 180 - to 280-bp segments using a Covaris bath sonicator (duty cycle, 10\%; intensity, 5; cycles per burst, 200;
3 min for $\left.25^{\circ} \mathrm{C}\right)$. A library was prepared and captured with an Agilent SureSelect Human All Exon V6 kit (Agilent Technologies, Inc.). The quality-passed library was sequenced on an Illumina HiSeq X Ten sequencing system (Illumina, Inc.). BWA software (http://bio-bwa.sourceforge.net/) was used to align raw data against the human reference genome (GRCh37/hg19). Single-nucleotide polymorphisms (SNPs), small insertions/deletions (indels) and quality recalibration were identified using GATK software (Genome Analysis Toolkit; www.broadinstitute.org/gatk). All variants were annotated using ANNOVAR (annovar.openbioinformatics. org/en/latest/). A raw Binary Base Call file was converted into a FASTQ file, $12 \mathrm{G}$ bases were obtained for each sample and the average yield was $\sim 16.3 \mathrm{~Gb}$ with an error rate of $<0.1 \%$.

Sanger sequencing. Two pairs of primers were synthesized by BGI Group to confirm the compound heterozygous variants of POC1A, according to GRCh37/hg19. The primers were as follows: chr3:52179936 forward, 5'-GGCCATCTCAGACCC ATTTA-3' and reverse, 5'-GAAAGGAGGTGTCTGGGTC A-3'; chr3:52159160 forward, 5'-TTCTGAGATGCAGCCATG AG-3' and reverse, 5'-CCTGGACTTGTCCCTGTTGT-3'. Polymerase chain reaction (PCR) amplification was performed using the genomic DNA as a template in a Goldstar ${ }^{\circledR}$ PCR kit (cat. no. CW0655M; CoWin Biosciences), according to the manufacturer's protocols. Sanger sequencing was conducted using a BigDye ${ }^{\circledR}$ Terminator v3.1 cycle sequencing kit (Applied Biosystems; Thermo Fisher Scientific, Inc.) according to the manufacturer's protocol. The amplified PCR products were purified and then run on an Applied Biosystems ${ }^{\mathrm{TM}} 3500$ series genetic analyzer (Thermo Fisher Scientific, Inc.). The detailed protocol for the primers is provided in Table I.

Diagnostic protocol. The diagnostic protocol of SOFT syndrome can be described as follows: i) Clinical signs by physical examination, including short stature, onychodysplasia, facial deformities and hypotrichosis $(3,5,7)$; ii) skeletal imaging changes by X-ray including short and thick long bones with irregular changes in metaphysis, short femoral neck and delayed ossification of carpal and vertebral bones $(1,5,6)$; iii) molecular genetic test to identify $P O C A 1$ pathogenic variants $(2,3)$.

Genetic findings. Given the poor response to $\mathrm{rhGH}$ and the patient's unusual facial appearance, exome sequencing was performed on all three family members. Only one gene (POC1A; NM_015426.5) with two variants (minor allele frequency <0.0001) remained in the patient: i) c.850_851insG in exon 8 , resulting in amino acid changes p.Glu284Glyfs*9 and ii) c.593_605delGTGGGACGTGCAT (deletion mutation) in exon 6, resulting in amino acid changes p.Ser198Metfs*10. For these two variants, one came from her father, and the other came from her mother (Fig. 2A-D). According to the American College of Medical Genetics and Genomics guidelines (14), these two variants are pathogenic and were confirmed through Sanger sequencing analysis (Fig. 2A-D).

Imaging observations. X-ray examinations were performed on the patient at age 4 (Fig. 3A and B) and 12 (Fig. 3C) months. The images demonstrated that the long bones of the limbs and 
Table I. PCR conditions for amplification of POC1 centriolar protein A exon 6 and exon 8 fragments.

\begin{tabular}{llllll}
\hline Exon & \multicolumn{1}{c}{ Variant } & Forward primer & Reverse primer & Product size (bp) & $\begin{array}{c}\text { Annealing } \\
\text { temperature }\left({ }^{\circ} \mathrm{C}\right)\end{array}$ \\
\hline 6 & c.593_605delGTGGGACGTGCAT & chr3:52159160-F & chr3:52159160-R & 530 & 62 \\
8 & c.850_851insG & chr3:52179936-F & chr3:52179936-R & 351 & 60 \\
\hline
\end{tabular}
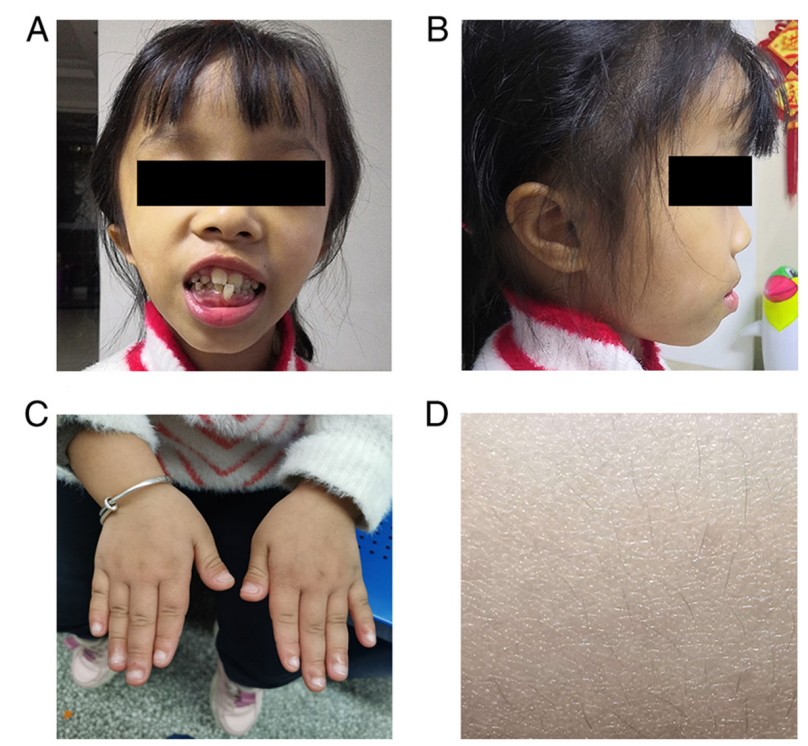

Figure 1. Phenotypic features of the patient. (A and B) Unusual facial features. (C) Hands with short and thick fingers, and hyperplasic nails. (D) Sparse body hair.
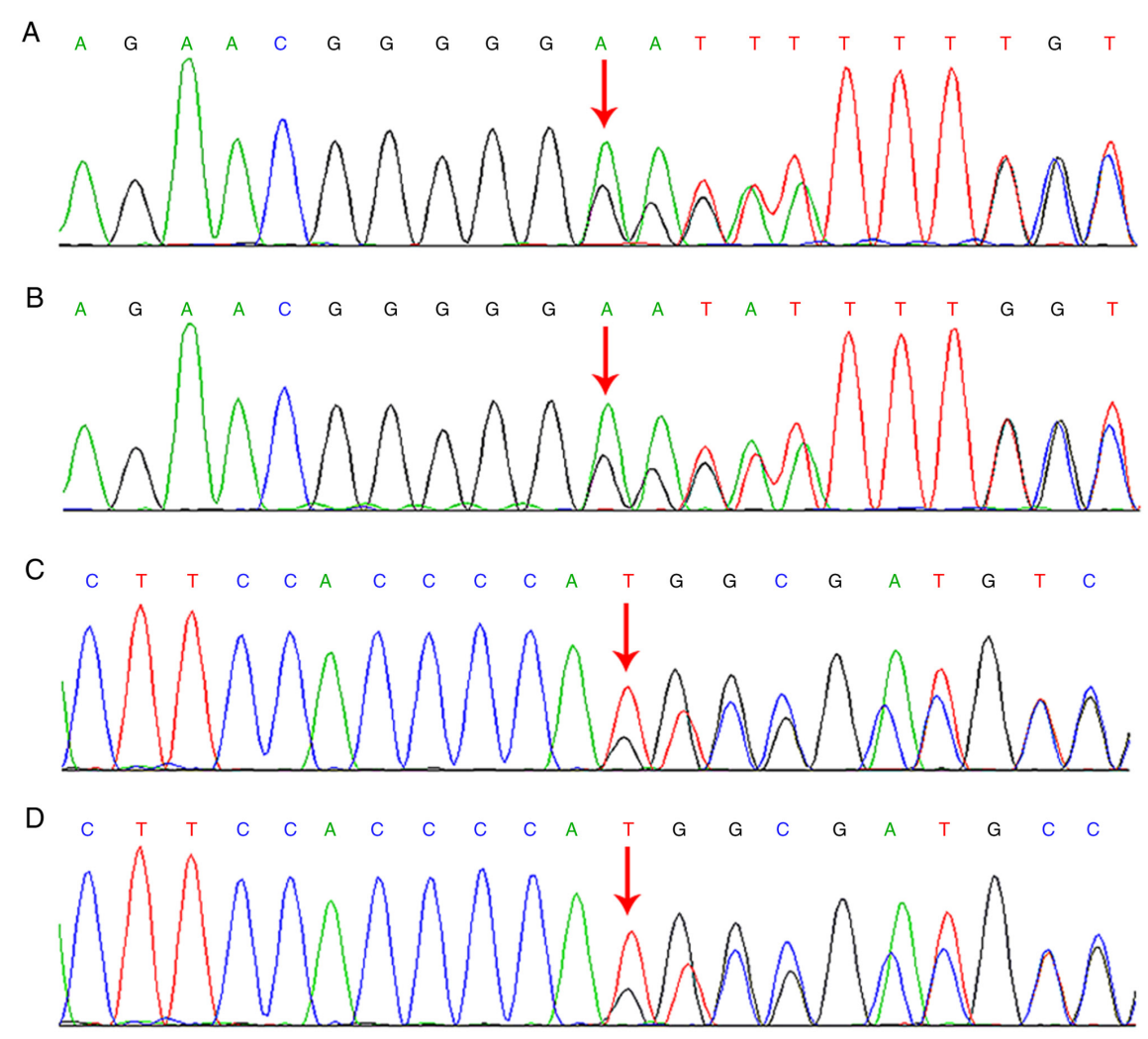

Figure 2. Sanger sequencing results of the patient and her parents. (A) Patient: Heterozygous variants of c.850_851insG on chr3:52159160. (B) Father: Heterozygous variants of c.850_851insG on chr3:52159160. (C) Patient: Heterozygous variants of c.593_605delGTGGGACGTGCAT on chr3:52179936. (D) Mother: Heterozygous variants of c.593_605delGTGGGACGTGCAT on chr3:52179936. 

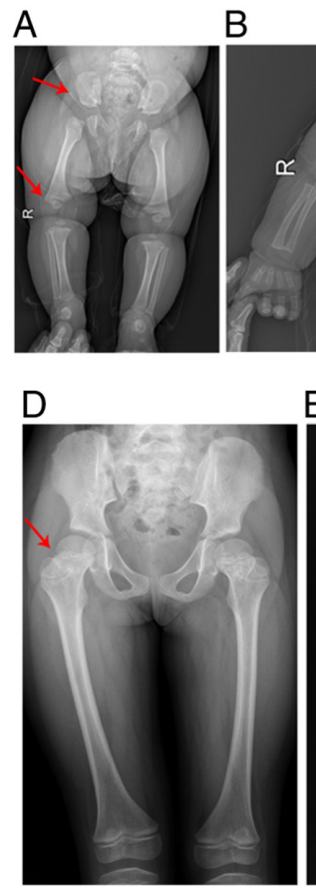

B

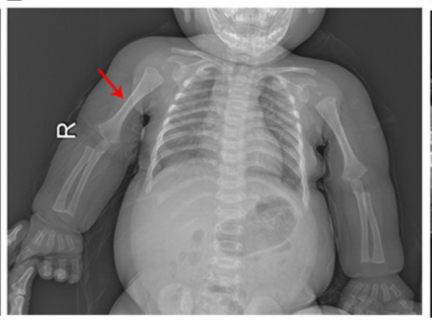

C

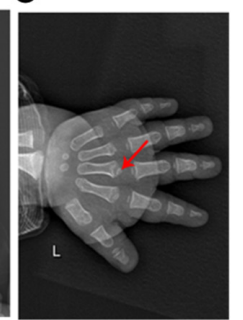

F

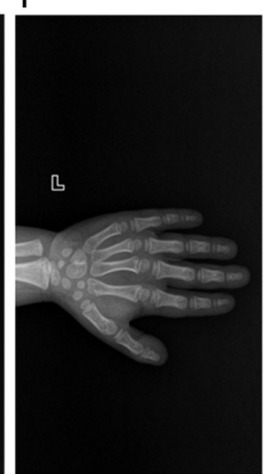

Figure 3. Skeletal X-ray examination of the patient. (A and B) The red arrows indicate shortened limb bones, widened metaphysis and an abnormal ilium at the age of 4 months. (C) The red arrow indicates shortened metacarpals and irregular metaphysis at the age of 1 year. (D and E) Shortened femoral neck (as indicated by the red arrow) and normal ilium, tibia and fibula at the age of 7 years. (F) Carpal bone at age 6.3 years with estimated bone age of $\sim 5.1$ years (according to the Tanner-Whitehouse 3 method).

A

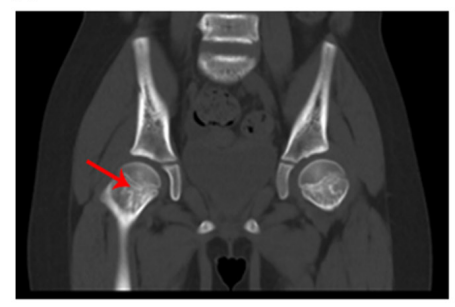

D

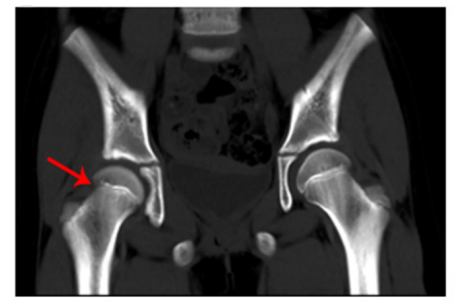

B

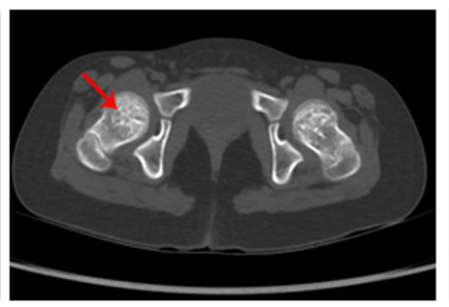

E

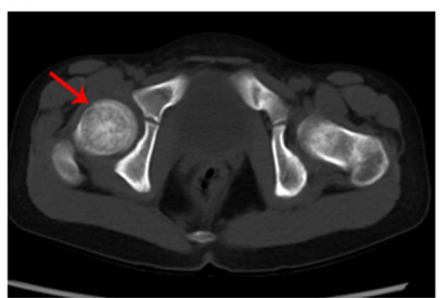

C

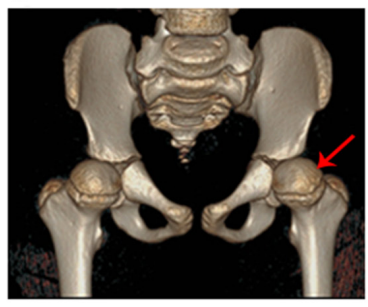

F

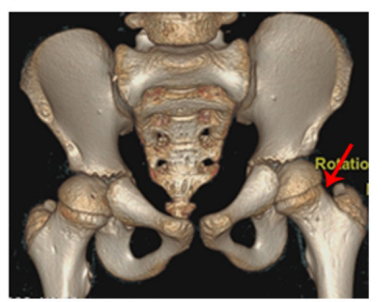

Figure 4. Spiral CT image of the hip. (A) Coronal and (B) transverse axis views demonstrate an uneven density (patchy high or low density) of the femoral neck as indicated by the red arrows. (C) Three-dimensional volume rendering revealed that the femoral neck was short and thick as indicated by the red arrow. (D-F) Normal hip spiral CT of an age- and sex-matched control. CT, computed tomography.

the metacarpal bones were thicker and shorter than those of an age- and gender-matched control. Furthermore, the metaphysis of these bones was widened, the ala of the ilium was square and the bottom of the ilium was shortened (Fig. 3A-C). However, when the patient was 7 years old (Fig. 3D), the dysplasia of these bones became less apparent and the malformation in the femur bones evolved into femur bones with a thicker and shorter femoral neck (Fig. 3D-F). Meanwhile, it was observed that when the patient was 6.3 years old, her carpal bone age was $\sim 5.1$ years old, according to the Tanner-Whitehouse 3 method (15) (Fig. 3F).
At the age of 7 years and 8 months, the patient was subjected to spiral computed tomography (CT) and the results showed that the bone density of the femoral neck was uneven (patchy high or low density) and that the femoral neck was thicker and shorter than those of the age- and gender-matched control (Fig. 4A-F). The three-dimensional double-echo steady state with water excitation MRI of the hip further revealed that the thickness of the proximal femoral epiphyseal plate was uneven and that the metaphysis of the proximal femur showed a stripe-like hyperintensity (such hyperintensity was continuous 

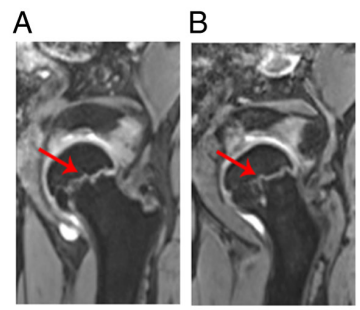

E

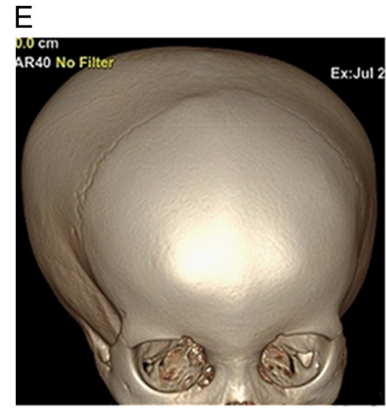

C

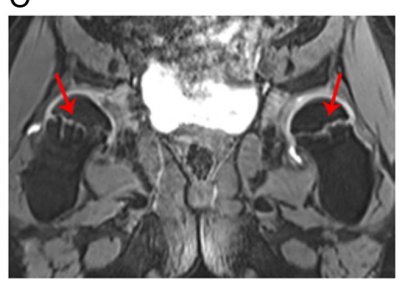

F

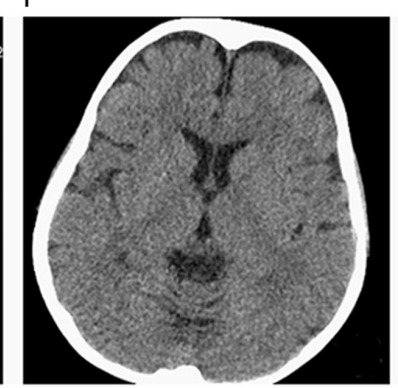

D

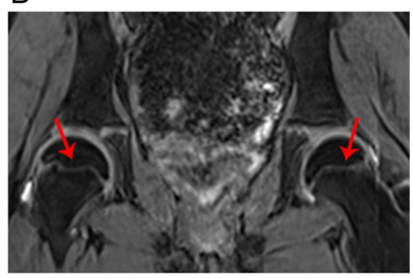

G

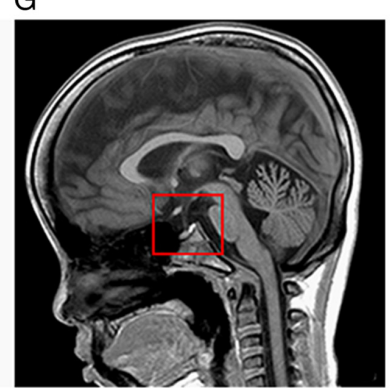

Figure 5. Three-dimensional double-echo steady state with water excitation MRI of the hip and imaging of the head. (A and B) Bilateral sagittal and (C) coronal views of the hip demonstrated that the thickness of the epiphyseal plate was uneven with stripe-like hyperintensity as indicated by the red arrow. (D) Normal hip MRI of the age- and sex-matched control in coronal view. (E) Three-dimensional volume rendering of the cranium presented trigonocephaly. (F) The brain computed tomography scan (transverse view) demonstrated the widened bilateral frontal extracerebral space. (G) T1-weighted image from the brain MRI (sagittal view). The red square indicates the flat sella. MRI, magnetic resonance imaging.

to the adjacent epiphyseal plate) (Fig. 5A-C). Fig. 5D shows the normal hip MRI of the age- and sex-matched control in coronal view. The three-dimensional volume rendering of the cranium identified the disproportionate cerebral and facial cranium as trigonocephaly, and the brain CT scan showed that the bilateral frontal extracerebral space was widened (Fig. 5E and F). The brain MRI indicated that the sella was flat and exhibited hook-like changes (Fig. 5G).

\section{Discussion}

Patients with SOFT syndrome exhibit numerous overlapping clinical features with several other syndromes, such as $3 \mathrm{M}$ syndrome, Russell-Silver syndrome and Mulibrey nanism. These clinical features include: i) Severe prenatal and postnatal growth retardation; ii) facial dysmorphism, including a triangular-shaped face and prominent forehead; and iii) normal intelligence (5). SOFT syndrome was diagnosed by the following features in the present case report: i) The patient had distinctive features, such as onychodysplasia, hypotrichosis and variable skeletal manifestations, including short long bones and irregular changes in metaphysis, which are consistent with the phenotypes of SOFT syndrome; ii) exome sequencing data did not reveal any rare variants on genes, as identified in 3M syndrome (CUL7, OBSL1, CCDC8) (16), Russell-Silver syndrome (GRB10, YWHAE) $(17,18)$ and Mulibrey nanism (TRIM37) (19); iii) exome sequencing identified two compound heterozygous variants of $P O C A 1$, which is the causative gene of SOFT syndrome.

POC1 consists of POC1A and POC1B (20). POC1A (located on 3p21.2) encodes POC1 centriolar protein A (2), which plays an important role in the early steps of centriole duplication and in the later phases of centriole length control, ensuring centriole integrity and proper mitotic spindle formation (20). POC1A and POC1B are conserved proteins, including an N-terminal WD40 domain, which likely forms a seven-bladed $\beta$-propeller and a $\mathrm{C}$-terminal coiled-coil, which contains a highly conserved sequence $(3,20,21)$. All of the POCA1 pathogenic variants reported to date disrupt the WD40 domains $(5-7,9,21)$. Consequently, the disruption interrupts the normal function of POC1 proteins and then causes the centrosome to fail to assemble; consequently, mitotic spindles form abnormally (5-7). In addition, the POC1A variants also cause the aberrant distribution of centrosomal microtubules $(3,7)$. Centrosomal microtubules play an essential role in proper Golgi assembly and trafficking, and several studies have shown a connection between the abnormal function of the Golgi and syndromes that feature bone and skin defects $(3,7)$. Therefore, POC1A plays an important role in the formation of bones and skin, and the dysfunction of centrosome formation may be the main molecular mechanism of SOFT syndrome (3). In the present study, according to the variants position at the nucleic acid level, the predicted amino acid changes in two POC1A variants are truncating from the WD40 domains. Therefore both variants of the present study may cause dysfunction of the centrosome, and the interruption of Golgi assembly and trafficking.

Shalev et al (1) investigated eight patients with SOFT syndrome in two families. Of these patients, three (ages 8, 15 and 25 years) received the skeletal imaging testing. Such three patients exhibited a severe to mild level of epiphyseal and metaphyseal changes in the long bones (metaphyseal changes are obvious on the 8 -year-old patient, but are mild on other older patients) (1). In the present study, the metaphysis abnormalities of the long bones and the hypoplasia of the ilium were clearly observed when the patient was 4- and 12-months-old, respectively. However, the dysplasia of these bones in the same patient was alleviated (or even disappeared) 
with aging. A skeletal X-ray of the patient at 7 years old identified that the metaphysis of the femur and metacarpal were becoming nearly normal. Moreover, the ilium dysplasia became less evident at this age. Therefore, similar to the X-ray examination results described by Shalev et al (1), the present results indicated that the metaphyseal dysplasia of the patient with SOFT syndrome was alleviated with aging. Investigating the underlying mechanism of the alleviation of metaphyseal and ilium dysplasia with age may provide insights into therapeutic strategies for SOFT syndrome in future.

Currently, the underlying mechanism of POC1A mutations in skeletal deformities remains unclear. In animal models with POC1A defects, certain studies implicated cell proliferation defects of chondrocytes in growth plates $(5,22)$. However, to the best of our knowledge, such proliferation defects have not been observed in humans. In the present study, the hip MRI revealed that the metaphysis of the femoral neck had a cartilaginous stripe signal and this finding was novel. This result indicated that an abnormal signal in the femoral neck may be the result of tissue imaging of immature chondrocytes during endochondral ossification. These imaging features provided evidence that the variant of $P O C I A$ was associated with the defect in the proliferation of chondrocytes in the growth plate; as a result, the growth of endochondral ossification was arrested. However, the detailed mechanism remains unclear; therefore, further studies are required to elucidate the function of POClA in endochondral ossification.

In conclusion, the present case report described a Chinese patient with SOFT syndrome caused by two novel POClA pathogenic variants, expanding the mutational and clinical spectrum of SOFT syndrome.

\section{Acknowledgements}

Not applicable.

\section{Funding}

No funding was received.

\section{Availability of data and materials}

The datasets generated and/or analyzed during the current study are available in the National Center for Biotechnology Information (NCBI) ClinVar repository, (https://www.ncbi. nlm.nih.gov/clinvar/variation/996335/; https://www.ncbi. nlm.nih.gov/clinvar/variation/996336/). The raw sequencing data generated and/or analyzed during the current study are not publicly available due to local government ethical restrictions and to protect the privacy of the family, but are available from the corresponding author on reasonable request.

\section{Authors' contributions}

YZ performed sample collection and clinical diagnosis, and reviewed and edited the manuscript. SL, YZ, YY, SH and WH wrote and edited the original manuscript. YY provided genetic guidance, helped to analyze the sequencing findings and reviewed the manuscript. SH oversaw imaging. YZ, WH and SL undertook sample collection and clinical data collection. All authors have read and approved the final manuscript. $\mathrm{YZ}$ and YY confirm the authenticity of all the raw data.

\section{Ethics approval and consent to participate}

The present study was approved by the Ethics Committee of Hunan Children's Hospital (Changsha, China).

\section{Patient consent for publication}

Prior to participating in this study, written informed consent was obtained from all three family members. Parents/guardians of both the patient and the matched control, as well as members of the family included in the present study, provided written informed consent for publication of the data and associated images in the present case report.

\section{Competing interests}

The authors declare that they have no competing interests.

\section{References}

1. Shalev SA, Spiegel R and Borochowitz ZU: A distinctive autosomal recessive syndrome of severe disproportionate short stature with short long bones, brachydactyly, and hypotrichosis in two consanguineous Arab families. Eur J Med Genet 55: 256-264, 2012.

2. Shaheen R, Faqeih E, Shamseldin HE, Noche RR, Sunker A, Alshammari MJ, Al-Sheddi T, Adly N, Al-Dosari MS, Megason SG, et al: POC1A truncation mutation causes a ciliopathy in humans characterized by primordial dwarfism. Am J Hum Genet 91: 330-336, 2012.

3. Sarig O, Nahum S, Rapaport D, Rapaport D, Ishida-Yamamoto A, Fuchs-Telem D, Qiaoli L, Cohen-Katsenelson K, Spiegel R, Nousbeck J, et al: Short stature, onychodysplasia, facial dysmorphism, and hypotrichosis syndrome is caused by a POC1A mutation. Am J Hum Genet 91: 337-342, 2012.

4. Al-Kindi A, Al-Shehhi M, Westenberger A, Beetz C, Scott P, Brandau O, Abbasi-Moheb L, Yüksel Z, Bauer P, Rolfs A and Grüning NM: A novel POC1A variant in an alternatively spliced exon causes classic SOFT syndrome: Clinical presentation of seven patients. J Hum Genet 65: 193-197, 2020

5. Koparir A, Karatas OF, Yuceturk B, Yuksel B, Bayrak AO, Gerdan OF, Sagiroglu MS, Gezdirici A, Kirimtay K, Selcuk E, et al: Novel POC1A mutation in primordial dwarfism reveals new insights for centriole biogenesis. Hum Mol Genet 24: 5378-5387, 2015 .

6. Ko JM, Jung S, Seo J, Shin CH, Cheong HI, Choi M, Kim OH and Cho TJ: SOFT syndrome caused by compound heterozygous mutations of POC1A and its skeletal manifestation. J Hum Genet 61: 561-564, 2016.

7. Barraza-García J, Iván Rivera-Pedroza C, Salamanca L, Belinchón A, López-González V, Sentchordi-Montané L, del Pozo Á, Santos-Simarro F, Campos-Barros Á, Lapunzina $\mathrm{P}$, et al: Two novel POC1A mutations in the primordial dwarfism, SOFT syndrome: Clinical homogeneity but also unreported malformations. Am J Med Genet A 170A: 210-216, 2016.

8. Saida K, Silva S, Solar B, Fujita A, Hamanaka K, Mitsuhashi S, Koshimizu E, Mizuguchi T, Miyatake S, Takata A, et al: SOFT syndrome in a patient from Chile. Am J Med Genet A 179: 338-340, 2019.

9. Mostofizadeh N, Gheidarloo M, Hashemipour M and Dehkordi EH: SOFT syndrome: The first case in Iran. Adv Biomed Res 7: 128, 2018.

10. Chen JH, Segni M, Payne F, Huang-Doran I, Sleigh A, Adams C; UK10K Consortium, Savage DB, O'Rahilly S, Semple RK and Barroso I: Truncation of POC1A associated with short stature and extreme insulin resistance. J Mol Endocrinol 55: 147-158, 2015. 
11. Giorgio E, Rubino E, Bruselles A, Pizzi S, Rainero I, Duca S, Sirchia F, Pasini B, Tartaglia M and Brusco A: A syndromic extreme insulin resistance caused by biallelic POC1A mutations in exon 10. Eur J Endocrinol 177: K21-K27, 2017.

12. Gong Y and Cai T: China revised the Webster's intelligence scale for children. Chin J Clin Psychol 2: 1-6, 1994.

13. Yang Y, Zheng Y, Li W, Li L, Tu M, Zhao L, Mei H, Zhu G and Zhu Y: SMAD6 is frequently mutated in nonsyndromic radioulnar synostosis. Genet Med 21: 2577-2585, 2019.

14. Richards S, Aziz N, Bale S, Bick D, Das S, Gastier-Foster J, Grody WW, Hegde M, Lyon E, Spector E, et al: Standards and guidelines for the interpretation of sequence variants: A joint consensus recommendation of the American college of medical genetics and genomics and the association for molecular pathology. Genet Med 17: 405-424, 2015.

15. Tanner JM, Healy MJR, Goldstein H and Cameron N: Assessment of skeletal maturity and prediction of adult height (TW3 method). London, Saunders, pp1-48, 2001.

16. Hu L, Wang X, Jin T, Han Y, Liu J, Jiang M, Yan S, Fu X, An B and Huang S: Identification of two CUL7 variants in two Chinese families with 3-M syndrome by whole-exome sequencing. J Clin Lab Anal 34: e23265, 2020.

17. Yoshihashi H, Maeyama K, Kosaki R, Ogata T, Tsukahara M, Goto Y, Hata J, Matsuo N, Smith RJ and Kosaki K: Imprinting of human GRB10 and its mutations in two patients with russell-silver syndrome. Am J Hum Genet 67: 476-482, 2000.

18. Ho AC, Liu AP, Lun KS, Tang WF, Chan KY, Lau EY, Tang MH, Tan TY and Chung BH: A newborn with a $790 \mathrm{~kb}$ chromosome 17 p13.3 microduplication presenting with aortic stenosis, microcephaly and dysmorphic facial features-is cardiac assessment necessary for all patients with $17 \mathrm{p} 13.3$ microduplication? Eur J Med Genet 55: 758-762, 2012.
19. Avela K, Lipsanen-Nyman M, Idänheimo N, Seemanová E, Rosengren S, Mäkelä TP, Perheentupa J, Chapelle AD and Lehesjoki AE: Gene encoding a new RING-B-box-Coiled-coil protein is mutated in mulibrey nanism. Nat Genet 25: 298-301, 2000.

20. Venoux M, Tait X, Hames RS, Straatman KR, Woodland HR and Fry AM: Poc1A and Poc1B act together in human cells to ensure centriole integrity. J Cell Sci 126: 163-175, 2013

21. Keller LC, Geimer S, Romijn E, Yates J III, Zamora I and Marshall WF: Molecular architecture of the centriole proteome: The conserved WD40 domain protein POC1 is required for centriole duplication and length control. Mol Biol Cell 20: 1150-1166, 2009

22. Geister KA, Brinkmeier ML, Burgess DL, Cavalcoli J, Cheung LY, Oatley JM, Oatley M, Wendt J and Camper SA: Pocla, a component of the centriole and cilia,causes skeletal dysplasia and male infertility: A mousemodel: American Society of Human Genetics Conference, San Diogo, California, USA, 2014.

This work is licensed under a Creative Commons Attribution-NonCommercial-NoDerivatives 4.0 International (CC BY-NC-ND 4.0) License. 